\title{
Wavelets and Wavelet Packets Applied to Termite Detection
}

\author{
Juan-José González de-la-Rosa ${ }^{1}$, Carlos García Puntonet ${ }^{2}$, \\ Isidro Lloret Galiana, and Juan Manuel Górriz \\ 1 University of Cádiz, EPS-Electronics Instrumentation Group, Electronics Area, Av. \\ Ramón Puyol S/N. 11202, Algeciras-Cádiz, Spain \\ juanjose.delarosa@uca.es \\ 2 University of Granada, Department of Architecture and Computers Technology, \\ ESII, C/Periodista Daniel Saucedo. 18071, Granada, Spain \\ carlos@atc.ugr.es
}

\begin{abstract}
In this paper we present an study which shows the possibility of using wavelets and wavelet packets to detect transients produced by termites. Identification has been developed by means of analyzing the impulse response of three sensors undergoing natural excitations. Denoising exhibits good performance up to $\mathrm{SNR}=-30 \mathrm{~dB}$, in the presence of white Gaussian noise. The test can be extended to similar vibratory or acoustic signals resulting from impulse responses.
\end{abstract}

\section{Introduction}

In acoustic emission (AE) signal processing a customary problem is to extract some physical parameters of interest in situations which involve join variations of time and frequency. This situation can be found in almost every nondestructive AE tests for characterization of defects in materials, or detection of spurious transients which reveal machinery faults [1. The problem of termite detection lies in this set of applications involving nonstationary signals [2].

When wood fibers are broken by termites they produce acoustic signals which can be monitored using ad hoc resonant AE piezoelectric sensors which include microphones and accelerometers, targeting subterranean infestations by means of spectral and temporal analysis. The drawbacks are the relative high cost and their practical limitations due to subjectiveness [2].

Second order methods (spectra) failure in low SNR conditions even with ad hoc piezoelectric sensors. Bispectrum have proven to be a useful tool for characterization of termites in relative noisy environments using low-cost sensors [3], 4]. The computational cost could be pointed out as the main drawback of the technique. This is the reason whereby diagonal bispectrum have to be used.

Numerous wavelet-theory-based techniques have evolved independently in different signal processing applications, like wavelets series expansions, multiresolution analysis, subband coding, etc. The wavelet transform is a well-suited technique to detect and analyze events occurring to different scales [5]. The idea of decomposing a signal into frequency bands conveys the possibility of extracting 
subband information which could characterize the physical phenomenon under study [6].

In this paper we show an application of wavelets' de-noising possibilities for the characterization and detection of termite emissions in low SNR conditions. Signals have been buried in Gaussian white noise. Working with three different sensors we find that the estimated signals' spectra match the spectra of the acoustic emission whereby termites are identified.

The paper is structured as follows: Section2 summarizes the problem of acoustic detection of termites; Section 3 remembers the theoretical background of wavelets and wavelet packets. Experiments and conclusions are drawn in Section 4.

\section{Acoustic Detection of Termites}

\subsection{Characteristics of the AE Signals}

Acoustic Emission(AE) is defined as the class of phenomena whereby transient elastic waves are generated by the rapid (and spontaneous) release of energy from a localized source or sources within a material, or the transient elastic wave(s) so generated (ASTM, F2174-02, E750-04, F914-0311).

Figure 1 shows one impulse in a burst produced by termites and its power spectrum. Significant drumming responses are produced over the range $200 \mathrm{~Hz}-$ $10 \mathrm{kHz}$. The carrier (main component) frequency of the drumming signal is around $2600 \mathrm{~Hz}$. The spectrum is not flat as a function of frequency as one would expect for a pulse-like event. This is due to the frequency response of the sensor (its selective characteristics) and also to the frequency-dependent attenuation coefficient of the wood and the air.

\subsection{Devices, Ranges of Measurement and HOS Techniques}

Acoustic measurement devices have been used primarily for detection of termites (feeding and excavating) in wood, but there is also the need of detecting termites in trees and soil surrounding building perimeters. Soil and wood have a much longer coefficient of sound attenuation than air and the coefficient increases with frequency. This attenuation reduces the detection range of acoustic emission to $2-5 \mathrm{~cm}$ in soil and $2-3 \mathrm{~m}$ in wood, as long as the sensor is in the same piece of material [7. The range of acoustic detection is much greater at frequencies $<10$ $\mathrm{kHz}$, and low frequency accelerometers have been used to detect insect larvae over 1-2 $\mathrm{m}$ in grain and $10-30 \mathrm{~cm}$ in soil [8].

It has been shown that ICA succeeded in separating termite emissions with small energy levels in comparison to the background noise. This is explained away by

\footnotetext{
${ }^{1}$ American Society for Testing and Materials. F2174-02: Standard Practice for Verifying Acoustic Emission Sensor Response. E750-04: Standard Practice for Characterizing Acoustic Emission Instrumentation. F914-03: Standard Test Method for Acoustic Emission for Insulated and Non-Insulated Aerial Personnel Devices Without Supplemental Load Handling Attachments
} 

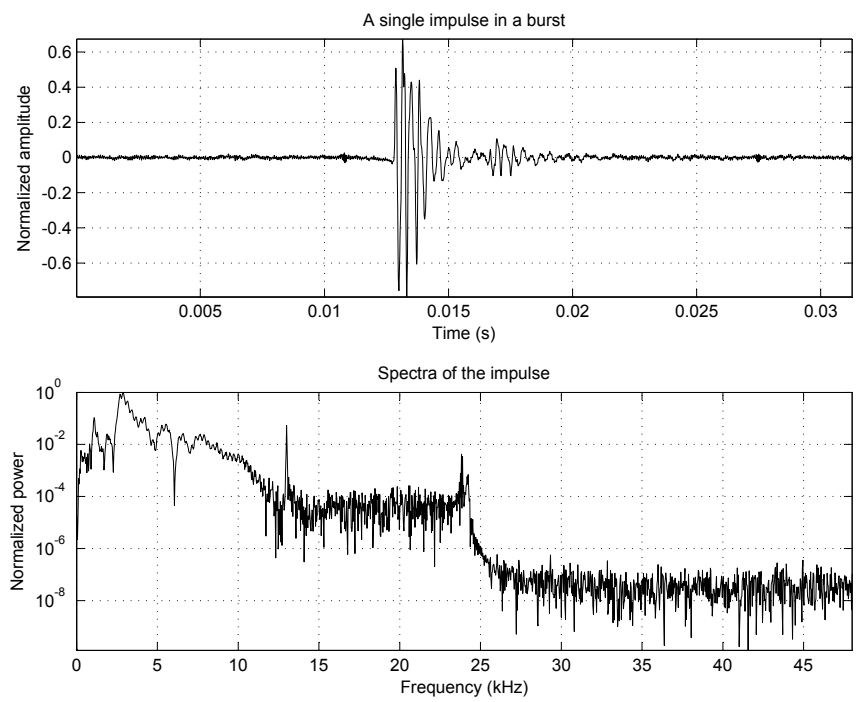

Fig. 1. Normalized power spectrum of a single pulse

statistical independence basis of ICA, regardless of the energy associated to each frequency component in the spectra 4. The same authors have proven that the diagonal bispectrum can be used as a tool for characterization purposes [3]. With the aim of reducing computational complexity wavelets transforms have been used in this paper to de-noise corrupted impulse trains.

\section{Wavelet Packets (WP)}

\subsection{Wavelet Bases}

The WP method is a generalization of wavelet decomposition that offers more possibilities of reconstructing the signal from the decomposition tree. If $L$ is the number of levels in the tree, WP methods yields more than $2^{2^{L-1}}$ ways to encode the signal. The wavelet decomposition tree is a part of the complete binary tree.

When performing a split we have to look at each node of the decomposition tree and quantify the information to be gained as a result of a split. An entropy based criterion is used herein to select the optimal decomposition of a given signal. We use an adaptative filtering algorithm, based on the work by Coifman and Wickerhauser 9.

Any finite energy signal $s(t)$ can be decomposed over a wavelet orthogonal basis [5] 2 of $\mathbf{L}^{2}(\Re)$ according to:

$$
s(t)=\sum_{j=-\infty}^{+\infty} \sum_{k=-\infty}^{+\infty}\left\langle s, \psi_{j, k}\right\rangle \psi_{j, k}
$$

$\overline{2\left\{\psi_{j, k}(t)=\frac{1}{\sqrt{2^{j}}} \psi\left(\frac{t-2^{j} k}{2^{j}}\right)\right\}_{(j, k) \in \mathbb{Z}^{2}}}$ 
Each partial sum can be interpreted as the details variations at the scale $a=2^{j}$ :

$$
d_{j}(t)=\sum_{k=-\infty}^{+\infty}\left\langle s, \psi_{j, k}\right\rangle \psi_{j, k} \quad s(t)=\sum_{j=-\infty}^{+\infty} d_{j}(t)
$$

The approximation of the signal $s(t)$ can be progressively improved by obtaining more layers or levels, with the aim of recovering the signal selectively. For example, if $s(t)$ varies smoothly we can obtain an acceptable approximation by means of removing fine scale details, which contain information regarding higher frequencies or rapid variations of the signal. This is done by truncating the sum in 1 at the scale $a=2^{J}$ :

$$
s_{J}(t)=\sum_{j=J}^{+\infty} d_{j}(t)
$$

\subsection{Multiresolution and Tree Decomposition}

We consider the resolution as the time step $2^{-j}$, for a scale $j$, as the inverse of the scale $2^{j}$. The approximation of a function $s$ at a resolution $2^{-j}$ is defined as an orthogonal projection on a space $\mathbf{V}_{j} \subset \mathbf{L}^{2}(\Re) . \mathbf{V}_{j}$ is called the scaling space and contains all possible approximations at the resolution $2^{-j}$.

Let us consider a scaling function $\phi$. Dilating and translating this function we obtain an orthonormal basis of $\mathbf{V}_{j}$ :

$$
\left\{\phi_{j, k}(t)=\frac{1}{\sqrt{2^{j}}} \phi\left(\frac{t-2^{j} k}{2^{j}}\right)\right\}_{(j, k) \in \mathbb{Z}^{2}} .
$$

The approximation of a signal $s$ at a resolution $2^{-j}$ is the orthogonal projection over the scaling subspace $\mathbf{V}_{j}$, and is obtained with an expansion in the scaling orthogonal basis $\left\{\phi_{j, k}\right\}_{k \in \mathbb{Z}}$ :

$$
P_{\mathbf{V}_{j}} s=\sum_{k=-\infty}^{+\infty}\left\langle s, \phi_{j, k}\right\rangle \phi_{j, k}
$$

The inner products

$$
a_{j}[k]=\left\langle s, \phi_{j, k}\right\rangle \phi_{j, k}
$$

represent a discrete approximation of the signal at level $j$ (scale $2^{j}$ ). This approximation is low-pass filtering of $s$ sampled at intervals $2^{-j}$.

A fast wavelet transform decomposes successively each approximation $P_{\mathbf{V}_{j-1}} s$ into a coarser approximation $P_{\mathbf{V}_{j}} s$ (local averages) plus the wavelet coefficients carried by $P_{\mathbf{W}_{j}} s$ (local details). The smooth signal plus the details combine into a multiresolution of the signal. Averages come from the scaling functions and details come from the wavelets.

$\left\{\phi_{j, k}\right\}_{k \in \mathbb{Z}}$ and $\left\{\psi_{j, k}\right\}_{k \in \mathbb{Z}}$ are orthonormal bases of $\mathbf{V}_{j}$ and $\mathbf{W}_{j}$, respectively, and the projections in these spaces are characterized by:

$$
a_{j}[k]=\left\langle s, \phi_{j, k}\right\rangle \quad d_{j}[k]=\left\langle s, \psi_{j, k}\right\rangle
$$




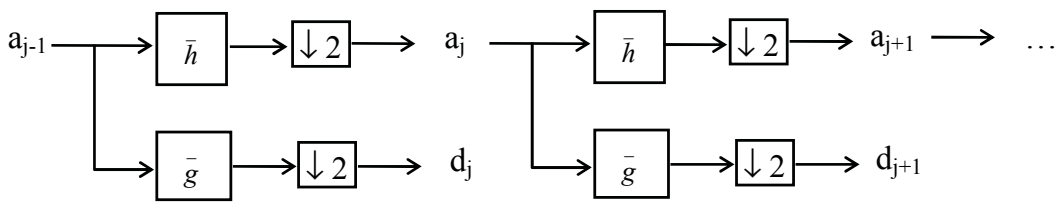

Fig. 2. Cascade of filters and subsampling

A space $\mathbf{V}_{j-1}$ is decomposed in a lower resolution space $\mathbf{V}_{j}$ plus a detail space $\mathbf{W}_{j}$, dividing the orthogonal basis of $\mathbf{V}_{j-1}$ into two new orthogonal bases:

$$
\left\{\phi_{j}\left(t-2^{j} k\right)\right\}_{k \in \mathbb{Z}} \quad \text { and } \quad\left\{\psi_{j}\left(t-2^{j} k\right)\right\}_{k \in \mathbb{Z}}
$$

$\mathbf{W}_{j}$ is the orthogonal complement of $\mathbf{V}_{j}$ in $\mathbf{V}_{j-1}$, and $\mathbf{V}_{j} \subset \mathbf{V}_{j-1}$, thus:

$$
\mathbf{V}_{j-1}=\mathbf{V}_{j} \oplus \mathbf{W}_{j}
$$

The orthogonal projection of a signal $s$ on $\mathbf{V}_{j-1}$ is decomposed as the sum of orthogonal projections on $\mathbf{V}_{j}$ and $\mathbf{W}_{j}$.

$$
P_{\mathbf{V}_{j-1}}=P_{\mathbf{V}_{j}}+P_{\mathbf{w}_{j}}
$$

The recursive splitting of these vector spaces is represented in the binary tree. This fast wavelet transform is computed with a cascade of filters $\bar{h}$ and $\bar{g}$, followed by a factor 2 subsampling, according with the scheme of figure 2 .

Functions that verify additivity-type property are suitable for efficient searching of the tree structures and node splitting. The criteria based on the entropy match these conditions, providing a degree of randomness in an informationtheory frame. In this work we used the entropy criteria based on the p-norm:

$$
E(s)=\sum_{i}^{N}\left\|s_{i}\right\|^{p} ;
$$

with $\mathrm{p} \leq 1$, and where $s=\left[s_{1}, s_{2}, \ldots, s_{N}\right]$ in the signal of length $N$. The results are accompanied by entropy calculations based on Shannon's criterion:

$$
E(s)=-\sum_{i}^{N} s_{i}^{2} \log \left(s_{i}^{2}\right)
$$

with the convention $0 \times \log (0)=0$.

\section{Experiments and Conclusions}

Two accelerometers (KB12V, seismic accelerometer; KD42V, industrial accelerometer, MMF) and a standard microphone have been used to collect data (alarm signals from termites) in different places (basements and subterranean wood structures and roots) using the sound card of a portable computer and a 

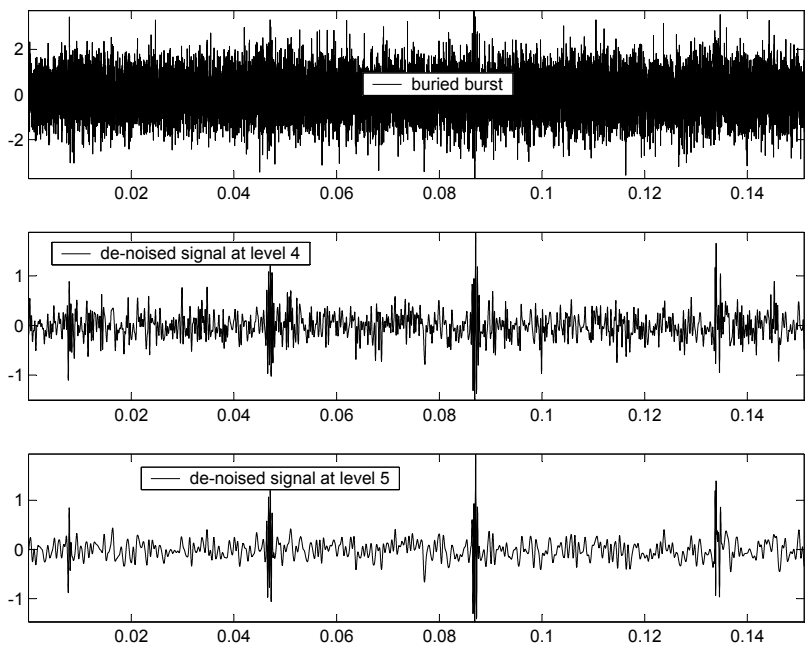

Fig. 3. Limit situation of the de-noising procedure using wavelets ( $\mathrm{SNR}=-30 \mathrm{~dB})$. From top to bottom: a buried 4-impulse burst, estimated signal at level 4, estimated signal at level 5

sampling frequency of $96000(\mathrm{~Hz})$, which fixes the time resolution. These sensors have different sensibilities and impulse responses. This is the reason whereby we normalize spectra.

The de-noising procedure was developed using a sym8, belonging to the family Symlets (order 8), which are compactly supported wavelets with least
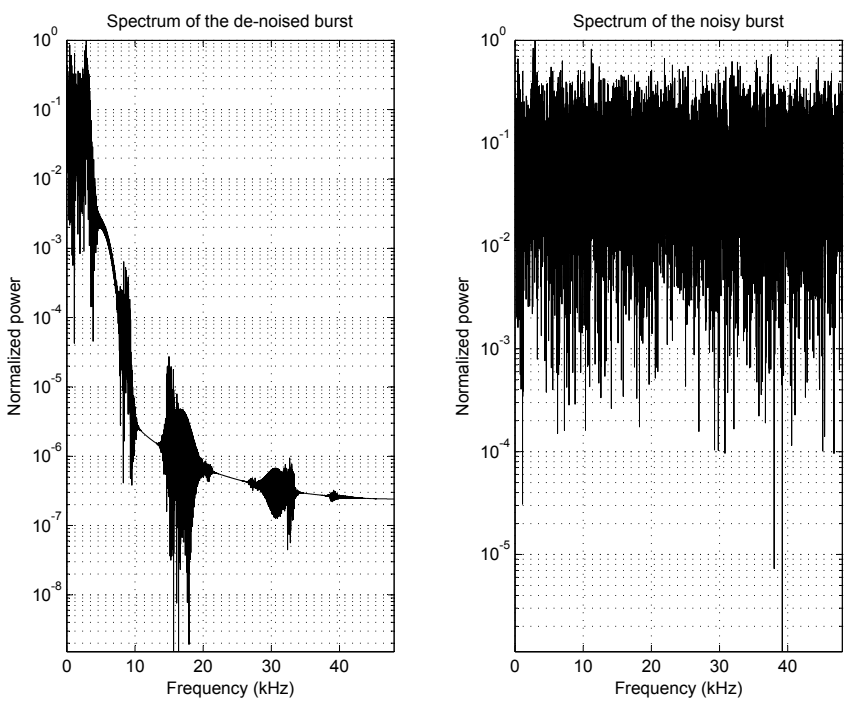

Fig. 4. Spectra of the estimated signal and the buried burst 

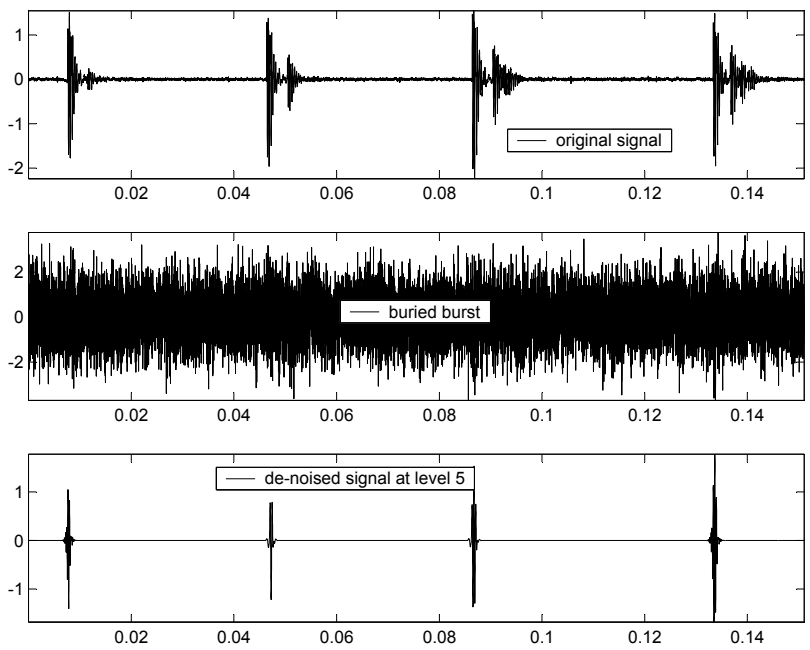

Fig. 5. Limit situation of the de-noising procedure using WP ( $\mathrm{SNR}=-30 \mathrm{~dB}$ ). From top to bottom: original signal, a buried 4-impulse burst, estimated signal at level 5

asymmetry and highest number of vanishing moments for a given support width. We also choose a soft heuristic thresholding.

We used 15 registers (from reticulitermes grassei), each of them comprises a 4-impulse burst buried in white gaussian noise. De-noising performs successfully up to an $\mathrm{SNR}=-30 \mathrm{~dB}$. Figure 3 shows a de-noising result in one of the registers. Figure 4 shows a comparison between the spectrum of the estimated signal at level 4 and the spectrum of the signal to be de-noised, taking a register as an example. Significant components in the spectrum of the recovered signal are found to be proper of termite emissions.

The same 15 registers were processed using wavelet packets. Approximation coefficients have been thresholded in order to obtain a more precise estimation of the starting points for each impulse. Stein's Unbiased Estimate of Risk (SURE) has been assumed as a principle for selecting a threshold to be used for denoising. A more thorough discussion of choosing the optimal decomposition can be found in [5]. Figure [5] shows one of the 15 de-noised signals using wavelets packets. It can be see the result of reconstructing progressively each $a_{j}$ by the filter banks.

\section{Acknowledgement}

The authors would like to thank the Spanish Ministry of Education and Science for funding the project DPI2003-00878, and the Andalusian Autonomous Government Division for funding the research with Contraplagas Ambiental S.L. 


\section{References}

1. Lou, X., Loparo, K.A.: Bearing fault diagnosis based on wavelet transform and fuzzy inference. Mechanical Systems and Signal Processing 18 (2004) 1077-1095

2. de la Rosa, J.J.G., Puntonet, C.G., Górriz, J.M., Lloret, I.: An application of ICA to identify vibratory low-level signals generated by termites. Lecture Notes in Computer Science (LNCS) 3195 (2004) 1126-1133 Proceedings of the Fifth International Conference, ICA 2004, Granada, Spain.

3. de la Rosa, J.G., Lloret, I., Puntonet, C.G., Górriz, J.M.: Higher-order statistics to detect and characterise termite emissions. Electronics Letters 40 (2004) 1316-1317 Ultrasosics.

4. de la Rosa, J.J.G., Puntonet, C.G., Lloret, I.: An application of the independent component analysis to monitor acoustic emission signals generated by termite activity in wood. Measurement 37 (2005) 63-76 Available online 12 October 2004.

5. Mallat, S.: A wavelet tour of signal processing. 2 edn. Academic Press (1999)

6. Angrisani, L., Daponte, P., D’Apuzzo, M.: A method for the automatic detection and measurement of transients. part I: the measurement method. Measurement $\mathbf{2 5}$ (1999) 19-30

7. Mankin, R.W., Osbrink, W.L., Oi, F.M., Anderson, J.B.: Acoustic detection of termite infestations in urban trees. Journal of Economic Entomology 95 (2002) 981-988

8. Robbins, W.P., Mueller, R.K., Schaal, T., Ebeling, T.: Characteristics of acoustic emission signals generated by termite activity in wood. In: Proceedings of the IEEE Ultrasonic Symposium. (1991) 1047-1051

9. Coifman, R.R., Wickerhauser, M.: Entropy-based algorithms for best basis selection. IEEE Trans. on Inf. Theory 38 (1992) 713-718 